EPJ Web of Conferences 33, 02011 (2012)

DOI: $10.1051 /$ epjconf/20123302011

(C) Owned by the authors, published by EDP Sciences, 2012

\title{
Ion motion in salt water flowing under a transverse magnetic field
}

\author{
R. De Luca
}

Università degli Studi di Salerno, Dipartimento di Fisica "E. R. Caianiello”, Fisciano (SA), Italy

\begin{abstract}
The problem of ion motion in an electrolyte solution flowing in a thin rectangular duct with velocity $\mathbf{V}_{\mathrm{E}}$ in the presence of a transverse magnetic field $\mathbf{B}_{0}$ is studied by means of classical mechanics and electrodynamics. Because of Lorentz force on the ions in the electrolyte solution, a so called Faraday voltage appears at the electrodes orthogonal to both the field $\mathbf{B}_{0}$ and the velocity $\mathbf{V}_{\mathrm{E}}$. The dynamics of positive and negative ions (cations and anions, respectively) in this classical system is studied by taking into account the viscosity of the fluid and the process of charge accumulation on the opposite walls of the duct. Hydrogen production is seen to take place at one of the electrodes when salt water is taken as the flowing electrolyte.
\end{abstract}

\section{Introduction}

Lorentz force acts on a charged particle moving in the presence of a magnetic field not parallel to the particle's velocity. In this way, conduction electrons flowing in a metal give rise to Hall effect [1], when they are subject to a transverse magnetic field. Similarly, we can consider anions and cations in an electrolyte solution flowing in a duct with velocity $\mathbf{v}=\mathbf{V}_{\mathrm{E}}+\mathbf{v}_{P, N}^{\prime}$, where $\mathbf{v}_{P, N}^{\prime}$ is the velocity of the particles measured with respect to a reference frame moving with the aqueous solution at velocity $\mathbf{V}_{E}$. In the presence of a constant magnetic field $\mathbf{B}_{0}$, these ions are subject to a Lorentz force given by

$$
\mathbf{F}_{P, N}= \pm Z e \mathbf{v} \times \mathbf{B}_{0}
$$

where the plus and minus signs refer to cations $(P)$ and anions $(N)$, respectively, both assumed to have ionic charge $Z e$ in absolute value ( $Z$ thus being the valence and $e$ the electronic charge in absolute value).

Because of Lorentz force, a voltage difference $f_{0}$, known in the literature as Faraday voltage [2, $3]$, appears between the electrodes $A$ and $B$ of fig. 1 . This voltage can be expressed by the product $B_{0} \mathrm{v}_{A} D$, when the electrolyte flows with an average velocity $\mathrm{v}_{A}$ in a cylindrical pipe of diameter $D$. In the present work boldface letters and corresponding plain text letters are used for vectors and their amplitude, respectively. Geselowitz [4] has shown that this result is valid for any velocity profile, provided the flow is axially symmetric. It can also be shown that this result is valid for a thin rectangular pipe [5].

\footnotetext{
a e-mail : rdeluca@unisa.it
} 
Aqueous ionic solutions and conducting metals show another similarity in their classical transport properties. In fact, one of the simplest phenomenological laws of electrodynamics is Ohm's law of conduction [1], namely:

$$
\mathbf{J}=\sigma \mathbf{E},
$$

where $\mathbf{J}$ is the current density flowing under the effect of the electric field $\mathbf{E}$, and $\sigma$ is the electrical conductivity of the material.

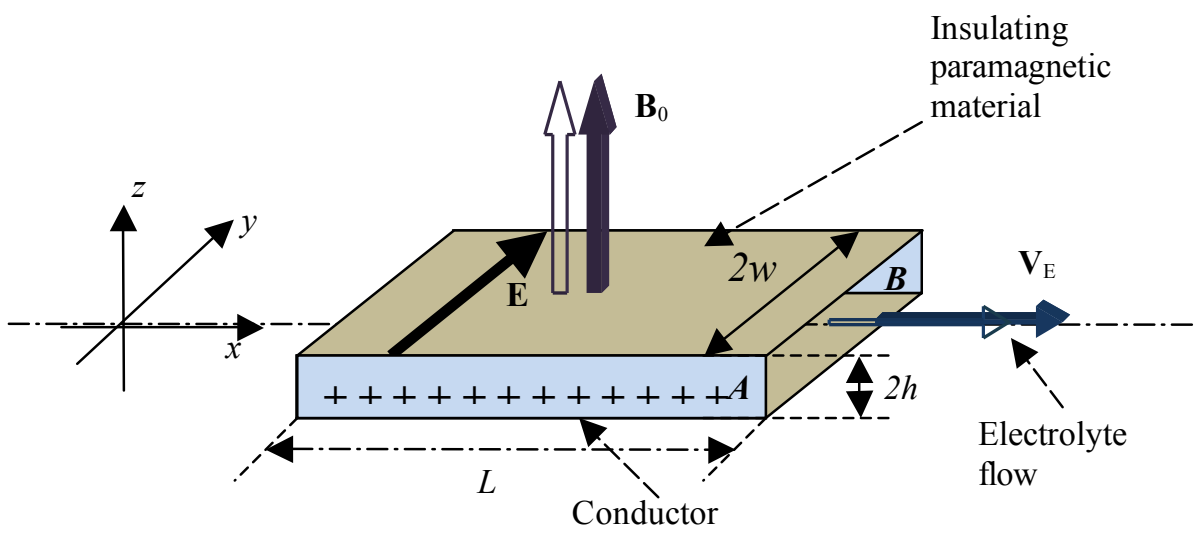

Fig. 1. An electrolyte solution flows through a rectangular pipe of section $4 w h$ ( $2 w$ is the width and $2 h$ the height) with velocity $\mathbf{V}_{\mathrm{E}}$ in a volume of length $L$ where a magnetic field $\mathbf{B}_{0}$, orthogonal to $\mathbf{V}_{\mathrm{E}}$, is present. Because of Lorentz force, the charged ions in the solution accumulate, in the vicinity of the lateral conducting plates, generating, at equilibrium, a constant electric field $\mathbf{E}_{0}$.

A similar relation can be found in electrolyte solutions. In the latter case, the partial conductivities, $\sigma_{P}$ and $\sigma_{N}$, can be expressed in terms of the mobility $\mu_{P}$ and $\mu_{N}$ of cations and anions, respectively, so that:

$$
\sigma_{P, N}=Z e \rho_{N} \mu_{P, N},
$$

where $\rho_{N}$ is the volume density of both types of ions giving rise to the current density $\mathbf{J}$. Mobility, on its turn, is defined as the proportionality constant linking the ions velocity $\mathbf{v}_{P, N}$ and the electric field $\mathbf{E}$, according to the following: $\mathbf{v}_{P, N}= \pm \mu_{P, N} \mathbf{E}$, where the plus and minus signs pertain to ions with homologous charge. We recall that the conductivity $\sigma$ is given by $\sigma=\sigma_{P}+\sigma_{N}$.

By starting with these simple notions, in the present work we shall approach the problem of ionic motion inside an electrolyte solution moving with velocity $\mathbf{V}_{\mathrm{E}}$ and subject to a magnetic field $\mathbf{B}_{0}$. A similar system has been already studied by Yamaguchi et al. [6] for a non-poisson electro-conductive polymer and its mixture with magnetic fluid. Similar studies on the dynamics of ionic charges flowing in an electrolyte flowing in the presence of a transverse magnetic field have been given in ref. [7]. In the present paper, however, results obtained by means of the proposed classical approach will be used in deriving the electrodynamic properties of the system.

Therefore, in the following section, the classical dynamical equations will be explicitly written. By solving these equations of the motion to first order in the relevant perturbation parameter, in the third section we are able to predict the behaviour of an electromotive force (e.m.f.) generator consisting of a rectangular pipe with two lateral conducting plates enclosing salt water in motion under a magnetic field orthogonal to the flow velocity vector (see fig. 1). In the fourth section, by taking salt water as the electrolyte flowing in the rectangular pipe, hydrogen gas is seen to be formed at one of the two electrodes of the e.m.f. generator proposed in the present work. The rate of 


\section{$2^{\text {nd }}$ European Energy Conference}

production of hydrogen gas as a function of the flux rate of salt water in the rectangular pipe is calculated. Applications of these types of systems are discussed in the last section.

\section{Ion motion}

Let us consider the schematic view of an electrolyte solution moving in a thin rectangular pipe in fig. 1. The velocity profile of the fluid in the pipe is taken to be uniform for simplicity. We would like to illustrate a simple dynamical model for ions moving in the electrolyte solution in the presence of a transverse magnetic field and a viscous force $\mathbf{R}_{P, N}=-\beta_{P, N} \mathbf{v}_{P, N}^{\prime}$, where $\beta_{P}$ and $\beta_{N}$ are the damping coefficients for cations and anions, respectively, and $\mathbf{v}_{P, N}^{\prime}$ represents the velocity of the positive $(P)$ and negative $(N)$ ions measured with respect to the frame moving with the aqueous solution at a velocity $\mathbf{V}_{\mathrm{E}}$. Moreover, given that charges accumulate on the two electrodes as in fig. 1, we also consider the presence of the electric field $\mathbf{E}=\left(0, E_{y}\right)$ in the system.

In this way, the total force on both species of ions will be given by

$$
\mathbf{F}_{P, N}= \pm Z e\left[\mathbf{E}+\left(\mathbf{V}_{\mathrm{E}}+\mathbf{v}_{P, N}^{\prime}\right) \times \mathbf{B}_{0}\right]
$$

where the plus and minus signs refer to cations $(P)$ and anions $(N)$, respectively. Because of the forces $\mathbf{F}_{P, N}$ and $\mathbf{R}_{P, N}$, the ions move relatively to the solvent according to the following classical equation of the motion

$$
\mathbf{F}_{P, N}+\mathbf{R}_{P, N}=m_{P, N} \mathrm{~d} \mathbf{v}_{P, N}^{\prime} / \mathrm{d} t
$$

where $m_{P}$ and $m_{N}$ are the masses of the positive $(P)$ and negative $(N)$ ions. By expressing $\mathbf{F}_{P, N}$ as in (4) and by setting $\mathbf{R}_{P, N}=-\beta_{P, N} \mathbf{v}_{P, N}^{\prime}$, we then have:

$$
\pm Z e\left[\mathbf{E}+\left(\mathbf{V}_{\mathrm{E}}+\mathbf{v}_{P, N}^{\prime}\right) \times \mathbf{B}_{0}\right]-\beta_{P, N} \mathbf{v}_{P, N}^{\prime}=m_{P, N} \mathrm{~d} \mathbf{v}_{P, N}^{\prime} / \mathrm{d} t,
$$

the plus and minus signs referring to cations $(P)$ and anions $(N)$, respectively. Notice that the only differences between the two equations of the motion, one written for positive ions, one for negative ions, resides on the different values of the masses and of the damping coefficients and on the sign in front of the square bracket in the first term in the left hand side and in the different signs in the relation $\mathbf{v}_{P, N}= \pm \mu_{P, N} \mathbf{E}$, written for the $y$-component. In this way, we may first solve the equation of motion for one species and successively obtain the solution for the second species just by changing the sign of the quantities $Z e$ and of the mobility, and by interchanging the subscripts $P$ and $N$. Let us then start by considering the dynamical equation (6) for cations. By setting $\mathbf{v}_{P}^{\prime}=\left(\mathrm{v}_{x}{ }^{\prime}, \mathrm{v}_{y}{ }^{\prime}\right)$, we write:

$$
\begin{gathered}
m_{P} \mathrm{dv}_{x}{ }^{\prime} / \mathrm{d} t=Z e B_{0} \mathrm{v}_{y}{ }^{\prime}-\beta_{P} \mathrm{v}_{x}{ }^{\prime}, \\
m_{P} \mathrm{dv}_{y}{ }^{\prime} / \mathrm{d} t=Z e\left[E_{y}-\left(\mathrm{v}_{x}{ }^{\prime}+\mathrm{V}_{E}\right) B_{0}\right]-\beta_{P} \mathrm{v}_{y}{ }^{\prime} .
\end{gathered}
$$

Notice now that $\mathrm{v}_{y}{ }^{\prime}=\mathrm{v}_{y}$ and $\mathrm{v}_{y}=\mu_{P} E_{y}$. In this way, by defining the constant $\alpha_{P}=Z e B_{0} / \beta_{P}$ and normalizing the laboratory time with respect to the constant $m_{P} / \beta_{P}$, we may rewrite (7a) and (7b) as follows:

$$
\begin{gathered}
\mathrm{dv}_{x}{ }^{\prime} / \mathrm{d} \tau+\mathrm{v}_{x}{ }^{\prime}=\alpha_{P} \mathrm{v}_{y} \\
\mathrm{dv}_{y} / \mathrm{d} \tau+\left(1-\alpha_{P} / \mu_{P} B_{0}\right) \mathrm{v}_{y}=-\alpha_{P} \mathrm{v}_{x}{ }^{\prime}-\alpha_{P} \mathrm{~V}_{E}
\end{gathered}
$$

where $\tau=\beta_{P} t / m_{P}$ is the normalized time. After some algebra, the above differential equation can be decoupled as follows:

$$
\mathrm{d}^{2} \mathrm{v}_{x}{ }^{\prime} / \mathrm{d} \tau^{2}+\left(2-\alpha_{P} / \mu_{P} B_{0}\right) \mathrm{dv}_{x}{ }^{\prime} / \mathrm{d} \tau+\left(1+\alpha_{P}{ }^{2}-\alpha_{P} / \mu_{P} B_{0}\right) \mathrm{v}_{x}{ }^{\prime}=-\alpha_{P}{ }^{2} \mathrm{~V}_{E},
$$




$$
\mathrm{d}^{2} \mathrm{v}_{y} / \mathrm{d} \tau^{2}+\left(2-\alpha_{P} / \mu_{P} B_{0}\right) \mathrm{dv}_{y} / \mathrm{d} \tau+\left(1+\alpha_{P}^{2}-\alpha_{P} / \mu_{P} B_{0}\right) \mathrm{v}_{y}=-\alpha_{P} \mathrm{~V}_{E}
$$

The stationary solutions of (9a) and (9b) are thus the following:

$$
\begin{aligned}
& \mathrm{v}_{x}{ }^{\prime}=-\alpha_{P}{ }^{2} \mathrm{~V}_{E} /\left(1+\alpha_{P}^{2}-\alpha_{P} / \mu_{P} B_{0}\right), \\
& \mathrm{v}_{y}=-\alpha_{P} \mathrm{~V}_{E} /\left(1+\alpha_{P}^{2}-\alpha_{P} / \mu_{P} B_{0}\right) .
\end{aligned}
$$

The dimensionless quantity $\alpha_{P}=Z e B_{0} / \beta_{P}$, on the other hand, is of the order of $10^{-7}$ for seawater in the presence of a magnetic field of 1.0 Tesla, so that, for any practical applications utilizing seawater or salt water with not much higher concentration of dilute ions, we can utilize a first-order solution for the solutions, finally setting:

$$
\begin{aligned}
& \mathrm{v}_{x}^{\prime} \approx 0, \\
& \mathrm{v}_{y} \approx-\alpha_{P} \mathrm{~V}_{E} .
\end{aligned}
$$

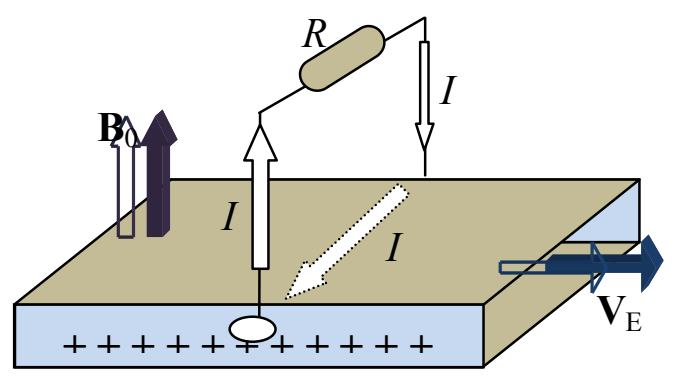

Fig. 2. A steady current $I$ flows through the resistor $R$ and the electrolyte solution flowing through a rectangular pipe of section $4 w h$ with velocity $\mathbf{V}_{\mathrm{E}}$.

Notice that, for what previously stated, in order to get the solution for negatively charged ions, it now suffices to change the sign in front of the parameter $\alpha_{P}$ in (11b) and to interchange $P$ with $N$. In this way, the relevant solutions to our problem are the following:

$$
\begin{aligned}
& \mathrm{v}_{y}^{(P)} \approx-\alpha_{P} \mathrm{~V}_{E}, \\
& \mathrm{v}_{y}{ }^{(N)} \approx+\alpha_{N} \mathrm{~V}_{E} .
\end{aligned}
$$

Moreover, we remark that the open-circuit solution to the problem is obtained by setting $E_{y}=E_{0}=$ $B_{0} \mathrm{~V}_{E}$ in (7b), so that the voltage across the device is $f_{0}=4 w B_{0} \mathrm{~V}_{E}$, where an extra factor of 2 , with respect to ref. [6], appears because of the presence of two ionic species. In order to derive the electrodynamic properties of the device, the strict implications of Eqs. (12a) and (12b) will be considered in the following section.

\section{The system as an electromotive force generator}

By considering that, under stationary conditions, the system may sustain a steady ionic flow in the $y$ direction, we can predict the steady current $I_{s c}$ can flow in the electrolyte, when the electrodes of the rectangular pipe are short-circuited, so that $R=0$ in fig. 2 . Noticing that the maximum current density $J_{y}$ is the sum of the cationic and anionic currents, we may write: 


$$
J_{y}=Z e \rho_{N}\left[\mathrm{v}_{y}^{(P)}-\mathrm{v}_{y}{ }^{(N)}\right]=-Z e \rho_{N}\left(\alpha_{P}+\alpha_{N}\right) \mathrm{V}_{E} .
$$

In the above expression, we have made explicit use of Eqs. (12a) and (12b). By now recalling that $\alpha_{P}=Z e B_{0} / \beta_{P}$ and that $\alpha_{N}=Z e B_{0} / \beta_{N}$, we may express $I_{s c}$ as follows:

$$
I_{s c}=2 h L(Z e)^{2} \rho_{N} B_{0} \mathrm{~V}_{E} / \beta_{e f f}=h L(Z e)^{2} \rho_{N} f_{0} / 2 w \beta_{e f f}
$$

where $\beta_{\text {eff }}=\beta_{P} \beta_{N} /\left(\beta_{P}+\beta_{N}\right)$. In the above expression the damping effects due to the viscous forces $\mathbf{R}_{P, N}$ are explicitly taken in to account. Let us now consider the Van der Waals radii $R_{P}$ and $R_{N}$ of cations and anions, respectively. By assuming that the damping coefficients $\beta_{P}$ and $\beta_{N}$ can be expressed in terms of the corresponding $R_{P}$ and $R_{N}$ values through Stokes' formula $\beta_{P, N}=6 \pi \eta R_{P, N}$, where $\eta$ is the viscosity of salt water, we may set:

$$
\beta_{e f f}=6 \pi \eta R_{e f f}
$$

where $R_{\text {eff }}=R_{P} R_{N} /\left(R_{P}+R_{N}\right)$.

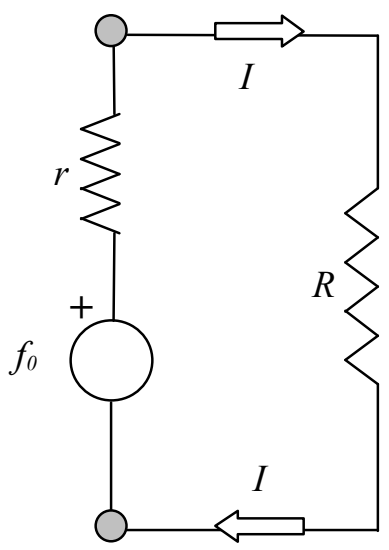

Fig. 3. Equivalent circuit of the system shown in fig. 1. The current $I$ flows through a resistor $R$ and inside the electrolyte solution having internal resistance $r$. The voltage across the ideal e. m. f. generator is $f_{0}=4 w B_{0} \mathrm{~V}_{E}$.

Therefore, by taking into account the predominant effect of the damping term, we may write the internal resistance of the device as follows:

$$
r=2 w \beta_{e f f} / h L(Z e)^{2} \rho_{N} .
$$

In this way the effective conductivity $\sigma_{e f f}$ may be expressed as

$$
\sigma_{e f f}=(Z e)^{2} \rho_{N} / 2 \beta_{e f f} .
$$

When we use the system as an e. m. f. generator, we would not be interested in driving short circuit current from it, but we would utilize it to provide useful power $P$ to an external load $R$.

$$
P=f_{0}^{2} R /(r+R)^{2}
$$

We drive the maximum power $P_{\max }$ out of the device when $R=r$, so that $P_{\max }=f_{0}^{2} / 4 r$.

\section{Hydrogen production}


The presence of a steady current $I$ in the system must be sustained by oxidation of chlorine ions and reduction of water at the two electrodes. In fact, at the negatively charged electrode production of chlorine gas occurs, according to the following reaction [8]:

$$
2 \mathrm{Cl}^{-} \rightarrow \mathrm{Cl}_{2}(g)+2 e^{-} .
$$

On the other hand, at the positively charged electrode reduction of water takes place according to the following [8]:

$$
2 \mathrm{Na}^{+}+2 e^{-}+2 \mathrm{H}_{2} \mathrm{O} \rightarrow 2 \mathrm{NaOH}+\mathrm{H}_{2}(g)+2 e^{-} .
$$

According to (20) production of sodium hydroxide and hydrogen gas takes place at the front electrode in fig. 2. Therefore, also confiding in the experimental evidence in ref. [1], we may argue that it is possible to devise an e.m.f. generator by letting salt water run in a pipe with velocity $\mathrm{V}_{E}$ in the presence of a magnetic field $\mathbf{B}_{0}$, orthogonal to the fluid flow. At the same time, hydrogen production takes place at one electrode of this particular e. m. f. generator.

By now considering that one mole of hydrogen is produced by the device when two moles of electrons are involved in the two reactions at both electrodes, we may write:

$$
\mathrm{dn}_{H} / \mathrm{d} t=I / 2 e N_{A},
$$

where $N_{A}$ is Avogadro's number. By now considering $I=I_{s c}$, the above expression can be expressed as follows:

$$
\mathrm{dn}_{H} / \mathrm{d} t==Z^{2} e B_{0} \rho_{N} h L \mathrm{~V}_{E} / N_{A} \beta_{e f f}
$$

Typical values of seawater concentrations at $25^{\circ} \mathrm{C}$ can be seen to be $n_{S W}=\rho_{N} / N_{A}=0.43 \mathrm{~mole} / \mathrm{l}=430$ mole $/ \mathrm{m}^{3}$. Therefore, by taking a flux rate $Q=4 w h \mathrm{~V}_{E}=10.0 \mathrm{l} / \mathrm{s}, w=0.2 \mathrm{~m}, L=0.5 \mathrm{~m}, B_{0}=2.0 \mathrm{~T}$, and by estimating $\beta_{\text {eff }}$ to be $1.72 \times 10^{-12} \mathrm{Kg} / \mathrm{s}$ through the knowledge of the Van der Waals' radii of the ionic species and the viscosity of seawater at $25^{\circ} \mathrm{C}$, we have that the maximum rate of hydrogen production in the device is approximately given by $\mathrm{dn}_{H} / \mathrm{d} t \approx 0.50 \times 10^{-6} \mathrm{~mole} / \mathrm{s}$. This result is of the same order of magnitude, though slightly lower than what reported in ref. [7], where the complete dynamics describing ion motion was not explicitly taken into account. When the system is made to work also as a power generator, so that a maximum power $P_{\max }=f_{0}^{2} / 4 r$ is driven out by making a current $I=I_{s c} / 2$ circulate in the circuit, the value of $P_{\max }$ is of the order of $10 \mathrm{~mW}$, being $f_{0}=0.4$ Volt, and the rate of hydrogen production is half the value calculated above.

\section{Conclusion}

We have predicted, by means of a classical model for ion motion in an electrolyte solution, that a steady transverse ionic current arises when a magnetic field is applied to an electrolyte flowing in a duct. If the system is not connected to an external circuit, a so called Faraday voltage appears at the two opposite electrodes placed along a direction orthogonal both to the electrolyte flow and the applied magnetic field. The conductivity of the solution is estimated in terms of the Van der Waals radii of the sodium and chlorine ions. When the electrodes are short-circuited, one notices that hydrogen gas is produced at one electrode with a rate $\mathrm{dn}_{H} / \mathrm{d} t$. Maximum power is driven out of the device when an external load of resistance $R$ equal to the internal resistance $r$ is added to the external circuit. In the latter case, half the maximum rate of hydrogen production is obtained.

Therefore, the proposed device may at the same time work as an e.m.f. generator and as an hydrogen production system. Being both the useful maximum power output $P_{\max }$ and the hydrogen production rate $\mathrm{dn}_{H} / \mathrm{d} t$ proportional to the ionic number density $\rho_{N}$, we may argue that by increasing the latter quantity, we may obtain higher values of both $P_{\max }$ and $\mathrm{dn}_{H} / \mathrm{d} t$. However, when the concentration of ions is increased, some of the assumption set forth in the above simple analysis 


\section{$2^{\text {nd }}$ European Energy Conference}

might result to be inadequate as, for instance, the independence of the velocity vector from the position along the y-axis. Finally, experimental work is needed to confirm the analysis carried out in the present work and further theoretical work is necessary to describe ion motion under more general conditions.

\section{References}

1. C. Kittel, Introduction to Solid State Physics (John Whiley, New York, 1959)

2. J. J. Wright and S. Van Der Beken, Am. J. Phys. 40, 245 (1972)

3. H. S. T. Driver, Am. J. Phys. 46, 1275 (1978)

4. D. B. Geselowitz, Am. J. Phys. 40, 1183 (1972)

5. R. De Luca, Eur. J. Phys. 30, 459-466 (2009)

6. H. Yamaguchi, Xin-Rong Zhang, S. Higashi, M. Li, J. Magn. Magn. Mat. 320, 1406 (2008)

7. R. De Luca, J. Mod. Phys. 2, 1115 (2011)

8. P. Mazzoldi, M. Nigro, C. Voci, Fisica (EdiSES, Naples 1998) 